\title{
Direct calculation of mutual information of distant regions
}

\author{
Noburo Shiba \\ KEK Theory Center, High Energy Accelerator Research Organization, \\ 1-1 Oho, Tsukuba, Ibaraki 305-0801, Japan \\ E-mail: shibn@yukawa.kyoto-u.ac.jp
}

Abstract: We consider the (Rényi) mutual information, $I^{(n)}(A, B)=S_{A}^{(n)}+S_{B}^{(n)}-S_{A \cup B}^{(n)}$, of distant compact spatial regions $\mathrm{A}$ and $\mathrm{B}$ in the vacuum state of a free scalar field. The distance $\mathrm{r}$ between $\mathrm{A}$ and $\mathrm{B}$ is much greater than their sizes $R_{A, B}$. It is known that $I^{(n)}(A, B) \sim C_{A B}^{(n)}\langle 0|\phi(r) \phi(0)| 0\rangle^{2}$. We obtain the direct expression of $C_{A B}^{(n)}$ for arbitrary regions $\mathrm{A}$ and $\mathrm{B}$. We perform the analytical continuation of $n$ and obtain the mutual information. The direct expression is useful for the numerical computation. By using the direct expression, we can compute directly $I(A, B)$ without computing $S_{A}, S_{B}$ and $S_{A \cup B}$ respectively, so it reduces significantly the amount of computation.

Keywords: Field Theories in Higher Dimensions, Lattice Quantum Field Theory

ArXiv ePrint: 1907.07155 


\section{Contents}

1 Introduction 1

2 The review of the operator method to compute the Rényi entropy 3

3 The expansion of the glueing operator 4

4 The (Rényi) mutual information of distant regions $\quad 7$

5 Massless free scalar field theory in $(d+1)$ dimension $\quad 9$

5.1 Lattice formulation 9

$\begin{array}{ll}5.2 \text { Numerical calculations } & 11\end{array}$

6 Conclusion and discussions 14

$\begin{array}{ll}\text { A Derivation of } 0 \leq \lambda_{i}<1 & 15\end{array}$

B The calculation of $F(n, a, b)$ in (4.6)

B.1 The calculation of $\sum_{k=0}^{n-1} \frac{1}{1-a \cos \left(\frac{2 \pi k}{n}\right)} \quad 16$

B.2 The calculation of $\sum_{k=0}^{n-1} \frac{1}{1-a \cos \left(\frac{2 \pi k}{n}\right)} \frac{1}{1-b \cos \left(\frac{2 \pi k}{n}\right)} \quad 17$

\section{Introduction}

The entanglement entropy in the quantum field theory plays important roles in many fields of physics including the string theory [1-13], condensed matter physics [14-17], lattice gauge theories [18, 19], cosmology [20], and the physics of the black hole [21-26]. The entanglement entropy is a useful quantity which characterizes quantum properties of given states.

For a given density matrix $\rho$ of the total system, the entanglement entropy of the subsystem $\Omega$ is defined as

$$
S_{\Omega}=-\operatorname{Tr} \rho_{\Omega} \ln \rho_{\Omega},
$$

where $\rho_{\Omega}=\operatorname{Tr}_{\Omega^{c}} \rho$ is the reduced density matrix of the subsystem $\Omega$ and $\Omega^{c}$ is the complement of $\Omega$. The Rényi entropy $S_{\Omega}^{(n)}$ is defined as

$$
S_{\Omega}^{(n)}=\frac{1}{1-n} \ln \operatorname{Tr} \rho_{\Omega}^{n}
$$

The limit $n \rightarrow 1$ coincides with the entanglement entropy $\lim _{n \rightarrow 1} S_{\Omega}^{(n)}=S_{\Omega}$.

In this paper, we consider the (Rényi) mutual information, $I^{(n)}(A, B)=S_{A}^{(n)}+S_{B}^{(n)}-$ $S_{A \cup B}^{(n)}$, of distant compact spatial regions $A$ and $B$ in the vacuum state of a free scalar 
field. The distance $r$ between $A$ and $B$ is much greater than their sizes $R_{A, B}$. It is known that [28], when $r \gg R_{A, B}$, the (Rényi) mutual information behaves as

$$
I^{(n)}(A, B) \sim C_{A B}^{(n)}\langle 0|\phi(r) \phi(0)| 0\rangle^{2},
$$

where $C_{A B}^{(n)}$ depends on the shapes of the regions $A$ and $B$. When both $A$ and $B$ are the spheres and the scalar field is massless, the coefficient $C_{A B}^{(n)}$ for a free scalar field was calculated analytically by Cardy [28]. However, it is difficult to calculate $C_{A B}^{(n)}$ analytically when both $A$ and $B$ are not the spheres or the scalar field is not massless. In this paper, we obtain the direct expression of $C_{A B}^{(n)}$ for arbitrary regions $A$ and $B$ in the vacuum state of a scalar field which has a general dispersion relation. We perform the analytical continuation of $n$ and obtain the mutual information $I(A, B)=\lim _{n \rightarrow 1} I^{(n)}(A, B)$. The direct expression is useful for the numerical computation. By using the direct expression, we can compute directly $I(A, B)$ without computing $S_{A}, S_{B}$ and $S_{A \cup B}$ respectively, so it reduces significantly the amount of computation.

We comment on the advantages of this direct expression over the conventional numerical computation by the real time formalism. Entanglement entropy in free scalar fields can be calculated numerically by the real time formalism [21, 22]. In order to calculate the coefficient $C_{A B}^{(n=1)}$ by the real time formalism, we have to plot the mutual information $I(A, B)$ as a function of $r$ and extract the coefficient [26]. So we have to calculate numerically $S_{A \cup B}$ many times to plot $I(A, B)$ as a function of r. On the other hand, in our method, we separate the $\mathrm{r}$ dependence of $I(A, B)$ analytically and obtain the direct expression of $C_{A B}^{(n=1)}$. So, it reduces significantly the amount of computation.

To obtain the direct expression of $C_{A B}^{(n)}$, we use the operator method to compute the Rényi entropy developed in [29]. This operator method is based on the idea that $\operatorname{Tr} \rho_{\Omega}^{n}$ is written as the expectation value of the local operator at $\Omega$. This idea was originally used to compute $I^{(n)}(A, B)$ in the vacuum state by Cardy [28], Calabrese et al. [30] and Headrick [31]. This idea was generalized to an arbitrary density matrix $\rho$ and the local operator was explicitly constructed in [29]. Cardy's work [28] was generalized (at least for the computation of the mutual information as opposed to the mutual Rényi information) for any CFT with a scalar in [38]. The next to leading terms in the long distance expansion of the mutual information in a free scalar theory was studied in [39]. The leading term for the mutual Rényi information for two widely separated identical compound systems in a free scalar theory was studied in [40].

The present paper is organized as follows. In section 2 , we review the operator method to compute the Rényi entropy developed in [29]. In section 3, we expand the glueing operator which plays the important role in the operator method to compute the (Rényi) mutual information. In section 4 , we compute the (Rényi) mutual information and obtain the direct expression of $C_{A B}^{(n)}$. In section 5, we apply our method to a massless free scalar field in $(d+1)$ dimension and perform numerical calculation for $d=2,3$. We show that our method reproduces the results computed by the conventional real time formalism. 


\section{The review of the operator method to compute the Rényi entropy}

We review the operator method to compute the Rényi entropy developed in [29]. We consider $n$ copies of the scalar fields in $(d+1)$ dimensional spacetime and the $j$-th copy of the scalar field is denoted by $\left\{\phi^{(j)}\right\}$. Thus the total Hilbert space, $H^{(n)}$, is the tensor product of the $n$ copies of the Hilbert space, $H^{(n)}=H \otimes H \cdots \otimes H$ where $H$ is the Hilbert space of one scalar field. We define the density matrix $\rho^{(n)}$ in $H^{(n)}$ as

$$
\rho^{(n)} \equiv \rho \otimes \rho \otimes \cdots \otimes \rho
$$

where $\rho$ is an arbitrary density matrix in $H$. We can express $\operatorname{Tr} \rho_{\Omega}^{n}$ as

$$
\operatorname{Tr} \rho_{\Omega}^{n}=\operatorname{Tr}\left(\rho^{(n)} E_{\Omega}\right),
$$

where

$$
\begin{aligned}
E_{\Omega}= & \int \prod_{j=1}^{n} \prod_{x \in \Omega} D J^{(j)}(x) D K^{(j)}(x) \exp \left[i \int d^{d} x \sum_{l=1}^{n} J^{(l+1)}(x) \phi^{(l)}(x)\right] \\
& \times \exp \left[i \int d^{d} x \sum_{l=1}^{n} K^{(l)}(x) \pi^{(l)}(x)\right] \times \exp \left[-i \int d^{d} x \sum_{l=1}^{n} J^{(l)} \phi^{(l)}\right],
\end{aligned}
$$

where $\pi(x)$ is a conjugate momenta of $\phi(x),[\phi(x), \pi(y)]=i \delta^{d}(x-y)$, and $J^{(j)}(x)$ and $K^{(j)}(x)$ exist only in $\Omega$ and $J^{(n+1)}=J^{(1)}$ and we normalize the measure of the functional integral as $\int D J^{(j)} \exp \left[i \int d^{d} x J^{(j)}(x) f(x)\right]=\prod_{x \in \Omega} \delta(f(x))$ where $f(x)$ is an arbitrary function. Notice that $\phi$ and $\pi$ in (2.3) are operators and the ordering is important. This operator $E_{\Omega}$ is called as the glueing operator. When $\rho$ is a pure state, $\rho=|\Psi\rangle\langle\Psi|$, the equation (2.2) becomes

$$
\operatorname{Tr} \rho_{\Omega}^{n}=\left\langle\Psi^{(n)}\left|E_{\Omega}\right| \Psi^{(n)}\right\rangle
$$

where

$$
\left|\Psi^{(n)}\right\rangle=|\Psi\rangle|\Psi\rangle \ldots|\Psi\rangle
$$

The useful property of the glueing operator for calculating the mutual information is the locality. When $\Omega=A \cup B$ and $A \cap B=\emptyset$,

$$
E_{A \cup B}=E_{A} E_{B} .
$$

From the locality (2.6), the mutual Rényi information in the vacuum state can be expressed as the correlation function of the glueing operators,

$$
\frac{\operatorname{Tr} \rho_{A \cup B}^{(n)}}{\operatorname{Tr} \rho_{A}^{(n)} \operatorname{Tr} \rho_{B}^{(n)}}=\frac{\left\langle 0^{(n)}\left|E_{A} E_{B}\right| 0^{(n)}\right\rangle}{\left\langle 0^{(n)}\left|E_{A}\right| 0^{(n)}\right\rangle\left\langle 0^{(n)}\left|E_{B}\right| 0^{(n)}\right\rangle} .
$$

We consider $(d+1)$ dimensional free scalar field theory. For free scalar fields, it is useful to represent the glueing operator $E_{\Omega}$ in (2.3) as the normal ordered operator. We decompose $\phi$ and $\pi$ into the creation and annihilation parts,

$$
\phi(x)=\phi^{+}(x)+\phi^{-}(x), \quad \pi(x)=\pi^{+}(x)+\pi^{-}(x),
$$


where

$$
\begin{array}{ll}
\phi^{+}(x)=\int \frac{d^{d} p}{(2 \pi)^{d}} \frac{1}{\sqrt{2 E_{p}}} a_{p} e^{i p x}, & \phi^{-}(x)=\left(\phi^{+}(x)\right)^{\dagger}, \\
\pi^{+}(x)=\int \frac{d^{d} p}{(2 \pi)^{d}}(-i) \sqrt{\frac{E_{p}}{2}} a_{p} e^{i p x}, & \pi^{-}(x)=\left(\pi^{+}(x)\right)^{\dagger},
\end{array}
$$

here $E_{p}$ is the energy and $\left[a_{p}, a_{p^{\prime}}^{\dagger}\right]=(2 \pi)^{d} \delta^{d}\left(p-p^{\prime}\right)$. The commutators of these operators are

$$
\begin{aligned}
& {\left[\phi^{+}(x), \phi^{-}(y)\right]=\langle 0|\phi(x) \phi(y)| 0\rangle=\int \frac{d^{d} p}{(2 \pi)^{d}} \frac{1}{2 E_{p}} e^{i p(x-y)} \equiv \frac{1}{2} W^{-1}(x, y),} \\
& {\left[\pi^{+}(x), \pi^{-}(y)\right]=\langle 0|\pi(x) \pi(y)| 0\rangle=\int \frac{d^{d} p}{(2 \pi)^{d}} \frac{E_{p}}{2} e^{i p(x-y)} \equiv \frac{1}{2} W(x, y),} \\
& {\left[\pi^{+}(x), \phi^{-}(y)\right]=\left[\pi^{-}(x), \phi^{+}(y)\right]=-\frac{i}{2} \delta^{d}(x-y),}
\end{aligned}
$$

where we have defined the matrices $W$ and $W^{-1}$ which has continuous indices $x, y$ in (2.10) and $W^{-1}$ is the inverse of $W . W$ and $W^{-1}$ are positive definite symmetric matrices. By using (2.10) and the Baker-Campbell-Hausdorff (BCH) formula $e^{X} e^{Y}=e^{[X, Y]} e^{Y} e^{X}, e^{X+Y}=$ $e^{-\frac{1}{2}[X, Y]} e^{X} e^{Y}$, for $[[X, Y], X]=[[X, Y], Y]=0$, we obtain

$$
\begin{aligned}
\exp \left[i \int d^{d} x J^{\prime} \phi\right] \exp \left[i \int d^{d} x K \pi\right] \exp \left[-i \int d^{d} x J \phi\right] \\
=: \exp \left[i \int d^{d} x\left(K \pi+\left(J^{\prime}-J\right) \phi\right)\right]: \\
\times \exp \left[\int d^{d} x d^{d} y\left(-\frac{1}{4} K(x) A(x, y) K(y)-\frac{1}{4}\left(J^{\prime}-J\right)(x) D(x, y)\left(J^{\prime}-J\right)(y)\right)\right. \\
\left.\quad-\int d^{d} x \frac{i}{2} K(x)\left(J^{\prime}+J\right)(x)\right]
\end{aligned}
$$

where : $O$ : means the normal ordered operator of $O$. From $(2.11)$ we can rewrite $E_{\Omega}$ in (2.3) as the normal ordered operator,

$$
E_{\Omega}=\int \prod_{j=1}^{n} \prod_{x \in \Omega} D J^{(j)}(x) D K^{(j)}(x): \exp \left[i \sum_{l=1}^{n} \int d^{d} x\left(\left(J^{(l+1)}-J^{(l)}\right) \phi^{(l)}+K^{(l)} \pi^{(l)}\right)\right]: \exp [-\tilde{S}],
$$

where $J^{(n+1)}=J^{(1)}$ and

$$
\begin{aligned}
\tilde{S} \equiv \sum_{l=1}^{n} & {\left[\int d^{d} x d^{d} y\left[\frac{1}{4} K^{(l)}(x) W(x, y) K^{(l)}(y)+\frac{1}{4}\left(J^{(l+1)}-J^{(l)}\right)(x) W^{-1}(x, y)\left(J^{(l+1)}-J^{(l)}\right)(y)\right]\right.} \\
& \left.+\frac{i}{2} \int d^{d} x K^{(l)}(x)\left(J^{(l+1)}+J^{(l)}\right)(x)\right] .
\end{aligned}
$$

\section{The expansion of the glueing operator}

We consider a complex scalar field $\phi$ because it is useful for later calculation. The mutual information of a real free scalar field can be obtained by dividing the mutual information 
of the complex free scalar field by 2 . Then, the glueing operator becomes

$$
\begin{aligned}
E_{\Omega}=\int \prod_{j=0}^{n-1} \prod_{x \in \Omega} D J^{(j)}(x) D K^{(j)}(x) & : \exp \left[i \sum _ { l = 0 } ^ { n - 1 } \int d ^ { d } x \left(\left(J^{(l+1)}-J^{(l)}\right) \phi^{(l) *}+K^{(l)} \pi^{(l) *}\right.\right. \\
& \left.\left.+\left(J^{(l+1) *}-J^{(l) *}\right) \phi^{(l)}+K^{(l) *} \pi^{(l)}\right)\right]: \exp [-\tilde{S}],
\end{aligned}
$$

where

$$
\begin{gathered}
\tilde{S} \equiv \sum_{l=0}^{n-1}\left[\int d^{d} x d^{d} y\left[\frac{1}{2} K^{(l)}(x) A(x, y) K^{(l) *}(y)+\frac{1}{2}\left(J^{(l+1)}-J^{(l)}\right)(x) D(x, y)\left(J^{(l+1) *}-J^{(l) *}\right)(y)\right]\right. \\
\left.+\frac{i}{2} \int d^{d} x\left(K^{(l)}(x)\left(J^{(l+1) *}+J^{(l) *}\right)(x)+K^{(l) *}(x)\left(J^{(l+1)}+J^{(l)}\right)(x)\right)\right] .
\end{gathered}
$$

For the free scalar field, it is useful to use the following Fourier transformation,

$$
f^{(l)}=\frac{1}{\sqrt{n}} \sum_{k=0}^{n-1} e^{2 \pi i k l / n} \tilde{f}^{(k)}
$$

where $f^{(l)}$ is an arbitrary n dimensional vector and $\tilde{f}^{(k)}$ is its Fourier transformation, i.e. (3.3) is the definition of the Fourier transformation. The Fourier transformation diagonalizes the glueing operator,

$$
E_{\Omega}=\prod_{k=0}^{n-1} E_{\Omega}^{(k)}
$$

where

$$
\begin{aligned}
E_{\Omega}^{(k)}= & \int \prod_{x \in \Omega} D \tilde{J}^{(k)}(x) D \tilde{K}^{(k)}(x): \exp \left[i Q^{(k)}\right]: \exp \left[-\tilde{S}^{(k)}\right] \\
Q^{(k)} \equiv & \int d^{d} x\left[\left(e^{2 \pi i k / n}-1\right) \tilde{J}^{(k)} \tilde{\phi}^{(k) *}+\left(e^{-2 \pi i k / n}-1\right) \tilde{J}^{(k) *} \tilde{\phi}^{(k)}+\tilde{K}^{(k)} \tilde{\pi}^{(k) *}+\tilde{K}^{(k) *} \tilde{\pi}^{(k)}\right] \\
\tilde{S}^{(k)} \equiv & \int d^{d} x d^{d} y\left[\frac{1}{2} \tilde{K}^{(k)}(x) A(x, y) \tilde{K}^{(k) *}(y)+\frac{1}{2}\left(1-\cos \left(\frac{2 \pi k}{n}\right)\right) \tilde{J}^{(k)}(x) D(x, y) \tilde{J}^{(k) *}(y)\right] \\
& +\frac{i}{2} \int d^{d} x\left(\left(e^{-2 \pi i k / n}+1\right) \tilde{K}^{(k)}(x) \tilde{J}^{(k) *}(x)+\left(e^{2 \pi i k / n}+1\right) \tilde{K}^{(k) *}(x) \tilde{J}^{(k)}(x)\right) .
\end{aligned}
$$

In order to expand : $\exp \left[i Q^{(k)}\right]:$ in $E_{\Omega}^{(k)}$, we define $\langle\ldots\rangle$ as

$$
\langle\ldots\rangle \equiv \frac{\int \prod_{x \in \Omega} D \tilde{J}^{(k)}(x) D \tilde{K}^{(k)}(x) \exp \left[-\tilde{S}^{(k)}\right] \ldots}{\int \prod_{x \in \Omega} D \tilde{J}^{(k)}(x) D \tilde{K}^{(k)}(x) \exp \left[-\tilde{S}^{(k)}\right]}
$$

where ... is an arbitrary function of $\tilde{J}^{(k)}$ and $\tilde{K}^{(k)}$. When $\Omega$ is a compact spatial region, we express $E_{\Omega}^{(k)}$ as a sum of the local operators at a conventionally chosen point $x_{0}$ inside 
$\Omega$. Thus, we expand $E_{\Omega}^{(k)}$ as

$$
\begin{aligned}
\frac{E_{\Omega}^{(k)}}{\left\langle 0\left|E_{\Omega}^{(k)}\right| 0\right\rangle} & =1-\frac{1}{2}\left\langle:\left(Q^{(k)}\right)^{2}:\right\rangle+\cdots \\
& =1-\left(2-2 \cos \left(\frac{2 \pi k}{n}\right)\right) \int d^{d} x d^{d} y\left\langle\tilde{J}^{(k)}(x) \tilde{J}^{(k) *}(y)\right\rangle: \tilde{\phi}^{(k) *}(x) \tilde{\phi}^{(k)}(y):+\cdots \\
& =1-: \tilde{\phi}^{(k) *}\left(x_{0}\right) \tilde{\phi}^{(k)}\left(x_{0}\right):\left(2-2 \cos \left(\frac{2 \pi k}{n}\right)\right) \int d^{d} x d^{d} y\left\langle\tilde{J}^{(k)}(x) \tilde{J}^{(k) *}(y)\right\rangle+\cdots
\end{aligned}
$$

In the last line in (3.9), we expanded the operator : $\tilde{\phi}^{(k) *}(x) \tilde{\phi}^{(k)}(y)$ : around $x_{0}$ and took it outside the integral because we consider the case that $\Omega$ is a compact spatial region and this expansion is useful for the later computation of the Rényi mutual information. In order to represent the Gauss integrals of $\tilde{K}^{(k)}$ and $\tilde{J}^{(k)}$, we will use the following matrix notation,

$$
\begin{aligned}
W(x, y) & =\left(\begin{array}{cc}
W\left(x_{\Omega}, y_{\Omega}\right) & W\left(x_{\Omega}, y_{\Omega^{c}}\right) \\
W\left(x_{\Omega^{c}}, y_{\Omega}\right) & W\left(x_{\Omega^{c}}, y_{\Omega^{c}}\right)
\end{array}\right) \equiv\left(\begin{array}{cc}
A & B \\
B^{T} & C
\end{array}\right) \\
W^{-1}(x, y) & =\left(\begin{array}{cc}
W^{-1}\left(x_{\Omega}, y_{\Omega}\right) & W^{-1}\left(x_{\Omega}, y_{\Omega^{c}}\right) \\
W^{-1}\left(x_{\Omega^{c}}, y_{\Omega}\right) & W^{-1}\left(x_{\Omega^{c}}, y_{\Omega^{c}}\right)
\end{array}\right) \equiv\left(\begin{array}{cc}
D & E \\
E^{T} & F
\end{array}\right)
\end{aligned}
$$

where $x_{\Omega\left(\Omega^{c}\right)}$ and $y_{\Omega\left(\Omega^{c}\right)}$ are the coordinates in $\Omega\left(\Omega^{c}\right)$, where $\Omega^{c}$ is the complement of $\Omega$.

In order to calculate $\left\langle\tilde{J}^{(k)}(x) \tilde{J}^{(k) *}(y)\right\rangle$, we perform the $\tilde{K}^{(k)}$ integral first,

$$
\begin{aligned}
& \int \prod_{x \in \Omega} D \tilde{K}^{(k)}(x) \exp \left[-\tilde{S}^{(k)}\right] \\
& =\frac{1}{\operatorname{det}\left(\frac{A}{2 \pi}\right)} \exp \left[-\int d^{d} x d^{d} y \tilde{J}^{(k) *}(x)\left(A^{-1}+D+\cos \left(\frac{2 \pi k}{n}\right)\left(A^{-1}-D\right)\right)(x, y) \tilde{J}^{(k)}(x)\right] .
\end{aligned}
$$

From (3.12), we obtain

$$
\begin{aligned}
\left\langle\tilde{J}^{(k)}(x) \tilde{J}^{(k) *}(y)\right\rangle & =\frac{\int \prod_{x \in \Omega} D \tilde{J}^{(k)}(x) D \tilde{K}^{(k)}(x) \exp \left[-\tilde{S}^{(k)}\right] \tilde{J}^{(k)}(x) \tilde{J}^{(k) *}(y)}{\int \prod_{x \in \Omega} D \tilde{J}^{(k)}(x) D \tilde{K}^{(k)}(x) \exp \left[-\tilde{S}^{(k)}\right]} \\
& =\left(A^{-1}+D+\cos \left(\frac{2 \pi k}{n}\right)\left(A^{-1}-D\right)\right)^{-1}(x, y) .
\end{aligned}
$$

In order to separate the $\mathrm{n}$ dependence of $\left\langle\tilde{J}^{(k)}(x) \tilde{J}^{(k) *}(y)\right\rangle$, we rewrite it as

$$
\left(A^{-1}+D+\cos \left(\frac{2 \pi k}{n}\right)\left(A^{-1}-D\right)\right)^{-1}=X\left(1-\cos \left(\frac{2 \pi k}{n}\right) Y\right)^{-1} X
$$

where

$$
\begin{array}{ll}
X \equiv\left(A^{-1}+D\right)^{-1 / 2}, & Y \equiv X\left(D-A^{-1}\right) X \\
Y=O^{T} \Lambda O, & \Lambda=\operatorname{diag}\left(\lambda_{i}\right)
\end{array}
$$

Thus we obtain

$$
\left(X\left(1-\cos \left(\frac{2 \pi k}{n}\right) Y\right)^{-1} X\right)_{i, j}=\sum_{l} Z_{l i} \frac{1}{1-\lambda_{l} \cos \left(\frac{2 \pi k}{n}\right)} Z_{l j}
$$


where $Z=O X$ and we discretized the space coordinates in order to regularize the scalar field. In the appendix A, we show that the range of the eigenvalues $\lambda_{i}$ is

$$
0 \leq \lambda_{i}<1
$$

Finally, when $\Omega$ is a compact spatial region, we obtain the expansion of $E_{\Omega}^{(k)}$ as

$$
\frac{E_{\Omega}^{(k)}}{\left\langle 0\left|E_{\Omega}^{(k)}\right| 0\right\rangle}=1-: \tilde{\phi}^{(k) *}\left(x_{0}\right) \tilde{\phi}^{(k)}\left(x_{0}\right): C_{\Omega}^{(k)}+\cdots
$$

where

$$
\begin{aligned}
C_{\Omega}^{(k)} & \equiv\left(2-2 \cos \left(\frac{2 \pi k}{n}\right)\right) \int d^{d} x d^{d} y\left\langle\tilde{J}^{(k)}(x) \tilde{J}^{(k) *}(y)\right\rangle \\
& =\left(2-2 \cos \left(\frac{2 \pi k}{n}\right)\right) \sum_{i} \sum_{j} \sum_{l} Z_{l i} \frac{1}{1-\lambda_{l} \cos \left(\frac{2 \pi k}{n}\right)} Z_{l j} \\
& \equiv\left(2-2 \cos \left(\frac{2 \pi k}{n}\right)\right) \sum_{i_{\Omega}} \sum_{j_{\Omega}} \sum_{l_{\Omega}} Z_{l_{\Omega} i_{\Omega}}^{(\Omega)} \frac{1}{1-\lambda_{l_{\Omega}}^{(\Omega)} \cos \left(\frac{2 \pi k}{n}\right)} Z_{l_{\Omega} j_{\Omega}}^{(\Omega)} .
\end{aligned}
$$

In the last line in (3.20), we added the subscript and the superscript $\Omega$ in order to clarify that $i_{\Omega}, j_{\Omega}$ and $l_{\Omega}$ are the coordinates in $\Omega$, and $Z^{(\Omega)}$ and $\lambda^{(\Omega)}$ depend on $\Omega$.

\section{The (Rényi) mutual information of distant regions}

We apply above results to the mutual Rényi information $I^{(n)}(A, B)$ of disjoint compact spatial regions $A$ and $B$ in the vacuum states of the free scalar field. From (2.7), (3.4) and (3.19), we obtain

$$
\begin{aligned}
\frac{\operatorname{Tr} \rho_{A \cup B}^{n}}{\operatorname{Tr} \rho_{A}^{n} \operatorname{Tr} \rho_{B}^{n}} & =\prod_{k=0}^{n-1} \frac{\left\langle 0\left|E_{A}^{(k)} E_{B}^{(k)}\right| 0\right\rangle}{\left\langle 0\left|E_{A}^{(k)}\right| 0\right\rangle\left\langle 0\left|E_{B}^{(k)}\right| 0\right\rangle} \\
& \simeq \prod_{k=0}^{n-1}\left\langle 0\left|\left(1-: \tilde{\phi}^{(k) *}\left(x_{A}\right) \tilde{\phi}^{(k)}\left(x_{A}\right): C_{A}^{(k)}\right)\left(1-: \tilde{\phi}^{(k) *}\left(x_{B}\right) \tilde{\phi}^{(k)}\left(x_{B}\right): C_{B}^{(k)}\right)\right| 0\right\rangle \\
& =\prod_{k=0}^{n-1}\left(1+C_{A}^{(k)} C_{B}^{(k)} f(r)\right)
\end{aligned}
$$

where $x_{A}$ and $x_{B}$ are some conventionally chosen points inside $A$ and $B, r=\left|x_{A}-x_{B}\right|$, and

$$
\begin{aligned}
f(r) & \left.\equiv\langle 0|: \tilde{\phi}^{(k) *}\left(x_{A}\right) \tilde{\phi}^{(k)}\left(x_{A}\right):: \tilde{\phi}^{(k) *}\left(x_{B}\right) \tilde{\phi}^{(k)}\left(x_{B}\right):\right)|0\rangle \\
& =\left(\left\langle 0\left|\phi\left(x_{A}\right) \phi^{*}\left(x_{B}\right)\right| 0\right\rangle\right)^{2}
\end{aligned}
$$

From (4.1), we obtain the mutual Rényi information as

$$
I^{(n)}(A, B)=\frac{1}{n-1} \ln \frac{\operatorname{Tr} \rho_{A \cup B}^{n}}{\operatorname{Tr} \rho_{A}^{n} \operatorname{Tr} \rho_{B}^{n}} \simeq \frac{1}{n-1} \sum_{k=0}^{n-1} \ln \left(1+C_{A}^{(k)} C_{B}^{(k)} f(r)\right) \simeq \frac{f(r)}{n-1} \sum_{k=0}^{n-1} C_{A}^{(k)} C_{B}^{(k)}
$$


We substitute $C_{\Omega}^{(k)}$ in (3.20) into (4.3) and obtain

$$
\begin{aligned}
I^{(n)}(A, B) & \simeq C_{A B}^{(n)} f(r), \\
C_{A B}^{(n)} & \equiv \frac{4}{n-1} \sum_{i_{A}} \sum_{j_{A}} \sum_{l_{A}} \sum_{i_{B}} \sum_{j_{B}} \sum_{l_{B}} Z_{l_{A} i_{A}}^{(A)} Z_{l_{A} j_{A}}^{(A)} Z_{l_{B} i_{B}}^{(B)} Z_{l_{B} j_{B}}^{(B)} F\left(n, \lambda_{l_{A}}^{(A)}, \lambda_{l_{B}}^{(B)}\right),
\end{aligned}
$$

where

$$
F(n, a, b) \equiv \sum_{k=0}^{n-1}\left(1-\cos \left(\frac{2 \pi k}{n}\right)\right)^{2} \frac{1}{1-a \cos \left(\frac{2 \pi k}{n}\right)} \frac{1}{1-b \cos \left(\frac{2 \pi k}{n}\right)} .
$$

We can perform explicitly the summation in (4.6) and obtain (see appendix B)

$$
\begin{aligned}
F(n, a, b)=n \frac{\left(1+p^{2}\right)\left(1+q^{2}\right)}{4 p q}\left[2-\frac{(1-p)\left(1+p^{n}\right)}{(1+p)\left(1-p^{n}\right)}-\frac{(1-q)\left(1+q^{n}\right)}{(1+q)\left(1-q^{n}\right)}\right. & \\
+\frac{2}{(1+p)(1+q)(p-q)(1-p q)} & \left\{\frac{1-p}{1-p^{n}} p(1-q)^{2}(1+q)-\frac{1-q}{1-q^{n}} q(1-p)^{2}(1+p)\right. \\
& -(p-q)(1+p q-(p+q) p q)\}]
\end{aligned}
$$

where

$$
p \equiv \rho(a)=\frac{1}{a}\left(1-\sqrt{1-a^{2}}\right), q \equiv \rho(b)=\frac{1}{b}\left(1-\sqrt{1-b^{2}}\right) .
$$

From (4.7), for $n=1,2,3$ and 4 , we obtain

$$
\begin{aligned}
& F(n=1, a, b)=0 \\
& F(n=2, a, b)=4 \frac{\left(1+p^{2}\right)}{(1+p)^{2}} \frac{\left(1+q^{2}\right)}{(1+q)^{2}} \\
& F(n=3, a, b)=\frac{9}{2} \frac{1+p^{2}}{1+p+p^{2}} \frac{1+q^{2}}{1+q+q^{2}} \\
& F(n=4, a, b)=2\left[1+2 \frac{\left(1+p^{2}\right)}{(1+p)^{2}} \frac{\left(1+q^{2}\right)}{(1+q)^{2}}\right] .
\end{aligned}
$$

When $n=2,3, F(n, a, b)$ is a product of the function of $a$ and $b$ and $C_{A B}^{(n)}$ becomes,

$$
C_{A B}^{(n)}=\tilde{C}_{A}^{(n)} \tilde{C}_{B}^{(n)}, \quad(n=2,3)
$$

where $\tilde{C}_{A(B)}^{(n)}$ is a function which is determined by the shape of $A(B)$. So, when $n=2,3$, $C_{A B}^{(n)}$ is not entangled, i.e. it is a simple product of functions each of which is determined by the shape of $A(B)$. In general, $F(n, a, b)$ is not a product of the function of $a$ and $b$ and $C_{A B}^{(n)}$ is entangled. However, we will compute $C_{A B}^{(n)}$ numerically later and show that $C_{A B}^{(n)}$ is factorized in a $(d+1)$ dimensional massless free scalar field for $d=2,3$. This factorization is not clear in the formal expressions in (4.7) and numerical calculation is important to check the factorization.

Because $F(n, a, b)$ is an elementary function of $n$, its analytical continuation is trivial. So we can take $n \rightarrow 1$ limit in $C_{A B}^{(n)}$ in (4.5). From (4.7) and (4.9), we obtain

$$
C_{A B}^{(n=1)}=\left.4 \sum_{i_{A}} \sum_{j_{A}} \sum_{l_{A}} \sum_{i_{B}} \sum_{j_{B}} \sum_{l_{B}} Z_{l_{A} i_{A}}^{(A)} Z_{l_{A} j_{A}}^{(A)} Z_{l_{B} i_{B}}^{(B)} Z_{l_{B} j_{B}}^{(B)}\left(\frac{\partial}{\partial n} F\left(n, \lambda_{l_{A}}^{(A)}, \lambda_{l_{B}}^{(B)}\right)\right)\right|_{n=1},
$$


where

$$
\left.\left(\frac{\partial}{\partial n} F(n, a, b)\right)\right|_{n=1}=\frac{1}{2} \frac{\left(1+p^{2}\right)\left(1+q^{2}\right)}{(1+p)(1+q)(p-q)(1-p q)}[(1-p)(1+q) \ln p-(1+p)(1-q) \ln q] .
$$

The calculation of the matrix $Z$ and the eigenvalues $\lambda_{i}$ is simple matrix computation. So, we can compute $C_{A B}^{(n=1)}$ numerically. Note that (4.4) is the mutual information of a free complex scalar field and the mutual information of a free real scalar field is a half of (4.4).

\section{Massless free scalar field theory in $(d+1)$ dimension}

\subsection{Lattice formulation}

We apply the above formalism to a massless free scalar field in $(d+1)$-dimensional Minkowski spacetime. The Lagrangian is given by

$$
L=\int d^{d} x \frac{1}{2}\left[\dot{\phi}^{2}-(\nabla \phi)^{2}\right]
$$

As an ultraviolet regulator, we replace the continuous $d$-dimensional space coordinates $x$ by a lattice of discrete points with spacing $a$. As an infrared cutoff, we allow the individual components of $n \equiv x / a$ to assume only a finite number $N$ of independent values $-N / 2<n_{\mu} \leq N / 2$. The Greek indices denoting vector quantities run from one to $d$. Outside this range we assume the lattice is periodic. The dimensionless Hamiltonian $H_{0} \equiv a H$ is given by

$$
H_{0} \equiv a H=\sum_{n}\left[\frac{1}{2} \pi_{n}^{2}+\frac{1}{2} \sum_{\mu=1}^{d}\left(\phi_{n_{\nu}+\delta_{\nu \mu}}-\phi_{n_{\nu}}\right)^{2}+\frac{a^{2} m^{2}}{2} \phi_{n}^{2}\right] \equiv \sum_{n} \frac{1}{2} \pi_{n}^{2}+\sum_{m, n} \frac{1}{2} \phi_{m} V_{m n} \phi_{n},
$$

where $\phi_{n}$ and $\pi_{n}$ are dimensionless and Hermitian, and obey the canonical commutation relations

$$
\left[\phi_{n}, \pi_{m}\right]=i \delta_{n m}
$$

In eq. (5.2) we insert a mass term in order to remove a zero eigenvalue of $V_{m n}$; if $V_{m n}$ should have the zero eigenvalue, $W^{-1}$ in (2.10) would not exist. Later we will take $N$ to infinity. In this limit we can neglect the zero eigenvalue of $V_{m n}$ and will take $a m$ to zero.

From (5.2) we obtain (see e.g. [27])

$$
\begin{aligned}
& W_{m n}=N^{-d} \sum_{k}\left[a^{2} m^{2}+2 \sum_{\mu=1}^{d}\left(1-\cos \frac{2 \pi k_{\mu}}{N}\right)\right]^{1 / 2} e^{2 \pi i k(n-m) / N}, \\
& W_{m n}^{-1}=N^{-d} \sum_{k}\left[a^{2} m^{2}+2 \sum_{\mu=1}^{d}\left(1-\cos \frac{2 \pi k_{\mu}}{N}\right)\right]^{-1 / 2} e^{2 \pi i k(n-m) / N},
\end{aligned}
$$

where the index $k$ also carries $d$ integer valued components, each in the range of $-N / 2<$ $k_{\mu} \leq N / 2$. We take $N$ to infinity and change the momentum sum into an integral with the 
replacements $q_{\mu}=2 \pi k_{\mu} / N$ and $N^{-d} \sum_{k} \rightarrow \int_{-\pi}^{\pi} \frac{d^{d} q}{(2 \pi)^{d}}$, and then we have

$$
\begin{aligned}
& W_{m n}=\int_{-\pi}^{\pi} \frac{d^{d} q}{(2 \pi)^{d}} e^{i q(n-m)}\left[a^{2} m^{2}+2 \sum_{\mu=1}^{d}\left(1-\cos q_{\mu}\right)\right]^{\frac{1}{2}}, \\
& W_{m n}^{-1}=\int_{-\pi}^{\pi} \frac{d^{d} q}{(2 \pi)^{d}} e^{i q(n-m)}\left[a^{2} m^{2}+2 \sum_{\mu=1}^{d}\left(1-\cos q_{\mu}\right)\right]^{\frac{-1}{2}} .
\end{aligned}
$$

In (5.6) and (5.7) the integrals converge when $a m \rightarrow 0$, so we can take $a m$ to zero,

$$
\begin{aligned}
& W_{m n}=\int_{-\pi}^{\pi} \frac{d^{d} q}{(2 \pi)^{d}} e^{i q(n-m)}\left[2 \sum_{\mu=1}^{d}\left(1-\cos q_{\mu}\right)\right]^{\frac{1}{2}}, \\
& W_{m n}^{-1}=\int_{-\pi}^{\pi} \frac{d^{d} q}{(2 \pi)^{d}} e^{i q(n-m)}\left[2 \sum_{\mu=1}^{d}\left(1-\cos q_{\mu}\right)\right]^{\frac{-1}{2}} .
\end{aligned}
$$

From (5.8) and (5.9) we can compute $W_{m n}$ and $W_{m n}^{-1}$ numerically. From (2.10) and (4.2), we obtain $f(r)=\frac{1}{4}\left(W^{-1}(x, y)\right)^{2}$. So we need to calculate $W_{m n}^{-1}$ for $\|n-m\| \gg 1$. The integrands in (5.8) and (5.9) highly oscillate when $\|n-m\| \gg 1$, and the numerical integrals converge very slowly. We can obtain approximate expressions of $W_{m n}$ and $W_{m n}^{-1}$ by hand when $\|n-m\| \gg 1[26]$. To evaluate $W_{m n}$ and $W_{m n}^{-1}$ when $\|n-m\| \gg 1$, we define $r \equiv a(n-m)$ and take $\|n-m\|$ to infinity keeping $r$ fixed. We change the variable as $p=q / a$, and then we have

$$
W_{m n}=a^{d} \int_{-\frac{\pi}{a}}^{\frac{\pi}{a}} \frac{d^{d} p}{(2 \pi)^{d}} e^{i p r}\left[2 \sum_{\mu=1}^{d}\left(1-\cos a p_{\mu}\right)\right]^{\frac{1}{2}} \rightarrow a^{d+1} \int_{-\infty}^{\infty} \frac{d^{d} p}{(2 \pi)^{d}} e^{i p r-\frac{a}{\pi}\|p\|}\left[\|p\|^{2}\right]^{\frac{1}{2}} .
$$

We can perform the integral in (5.10) analytically when $\|r\| / a \rightarrow \infty$ (see appendix A of [25]), and then we obtain

$$
W_{m n} \rightarrow a^{d+1} \frac{A_{d}}{\|r\|^{d+1}}=\frac{A_{d}}{\|n-m\|^{d+1}},
$$

where

$$
A_{d}= \begin{cases}-\frac{(d-1) ! !}{(2 \pi)^{d / 2}} & \text { for even } d \geq 2, \\ -\frac{(d-1) ! !}{(2 \pi)^{(d+1) / 2}} & \text { for odd } d \geq 3 .\end{cases}
$$

We can evaluate $W_{m n}^{-1}$ when $\|n-m\| \gg 1$ in the same way (see appendix A of [25] ), and then we obtain

$$
W_{m n}^{-1} \rightarrow a^{d-1} \int_{-\infty}^{\infty} \frac{d^{d} p}{(2 \pi)^{d}} e^{i p r-\frac{a}{\pi}\|\vec{p}\|}\left[\|\vec{p}\|^{2}\right]^{\frac{-1}{2}} \rightarrow a^{d-1} \frac{B_{d}}{\|r\|^{d-1}}=\frac{B_{d}}{\|n-m\|^{d-1}} .
$$




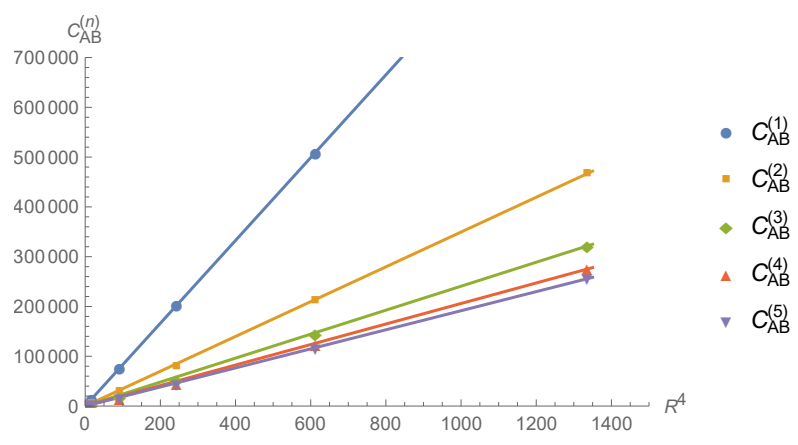

Figure 1. The coefficient $C_{A B}^{(n)}$ as a function of $R^{4}$, where $C_{A B}^{(n)}$ is the coefficient of the (Rényi) mutual information $I^{(n)}(A, B)$ of two spheres $A$ and $B$ whose radii are $R_{A}=R_{B} \equiv R$. The lines are the best linear fit.

where

$$
B_{d}= \begin{cases}\frac{(d-3) ! !}{(2 \pi)^{d / 2}} & \text { for even } \quad d \geq 2 \\ 2 \frac{(d-3) ! !}{(2 \pi)^{(d+1) / 2}} & \text { for odd } d \geq 3\end{cases}
$$

where $0 ! !=(-1) ! !=1$.

From (2.10), (4.2), (4.4) and (5.13), we obtain

$$
I^{(n)}(A, B) \simeq C_{A B}^{(n)} \frac{B_{d}^{2}}{4\|n-m\|^{2 d-2}},
$$

where $C_{A B}^{(n)}$ is defined in (4.5). Note that the lattice spacing $a$ is canceled out because the (Rényi) mutual information is dimensionless. So we calculate $C_{A B}^{(n)}$ in (5.15) by using the dimensionless matrices $W_{m n}$ and $W_{m n}^{-1}$.

\subsection{Numerical calculations}

We calculate numerically the (Rényi) mutual information $I^{(n)}(A, B)$ of two spheres $A$ and $B$ whose radii are $R_{A}=R_{B} \equiv R$, and the distance between the centers of them is $r$ for $d=3$. We put the centers of the spheres on a lattice. We define the sphere as a set of points which are at distances of $\tilde{R}$ or less from the center of the sphere. In order to reduce the effect of the discretization, we correct $\tilde{R}$ and define the radius of this sphere as $R:=(3 V / 4 \pi)^{1 / 3}$ where $V$ is a number of points in the sphere.

We show the computed values of $C_{A B}^{(n)}$ in (5.15) as a function of $R^{4}$ in figure 1. As can be seen, $C_{A B}^{(n)}$ is proportional to $R^{4}$.

Thus, we obtain

$$
I^{(n)}(A, B) \simeq g_{A B}^{(n)} \frac{R^{4}}{r^{4}}
$$

where $g_{A B}^{(n)}$ is a dimensionless constant. We calculate the coefficient $g_{A B}^{(n)}$ from slopes of graphs of $C_{A B}^{(n)}$ as a function of $R^{4}$. We show the computed values of $g_{A B}^{(n)}$ as a function of $n$ in figure 2 . 


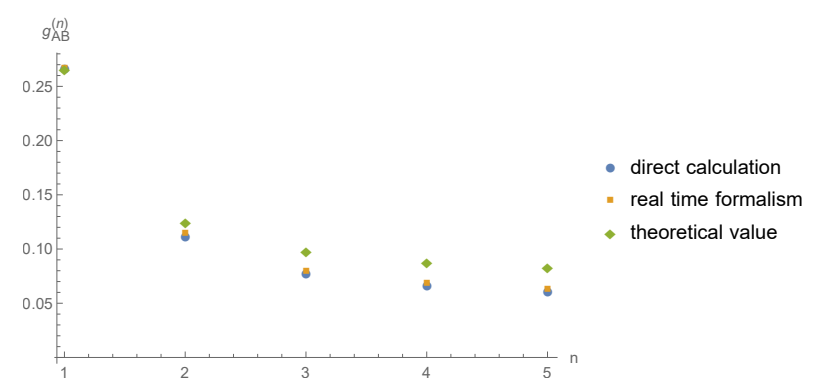

Figure 2. The coefficient $g_{A B}^{(n)}$ in (5.16) as a function of $n$. We show the values of $g_{A B}^{(n)}$ computed by the direct calculation developed in this paper and the real time formalism, and the theoretical values $\left.g_{A B}^{(n)}\right|_{d=3}=\frac{n^{4}-1}{15 n^{3}(n-1)}$.

For comparison, we also showed the values of $g_{A B}^{(n)}$ computed by the real time formalism [21] and the theoretical values $\left.g_{A B}^{(n)}\right|_{d=3}=\frac{n^{4}-1}{15 n^{3}(n-1)}$ obtained by Cardy [28]. In order to calculate the coefficient $g_{A B}^{(n)}$ by the real time formalism, we plotted the (Rényi) mutual information $I^{(n)}(A, B)$ as a function of $r$ and extract the coefficient in the same way as the mutual information [26]. From figure 2, for $n=1$, the value of $g_{A B}^{(n)}$ computed by the direct calculation and one computed by the real time formalism and theoretical one are almost equal. For $n>1$, the values of $g_{A B}^{(n)}$ computed by the direct calculation and those computed by the real time formalism are almost equal, however, the theoretical values $\left.g_{A B}^{(n)}\right|_{d=3}$ are slightly larger than numerical computed values. The discrepancy for $n>1$ probably indicates that the effect of the discretization become important when $n$ increases. The direct calculation of the (Rényi) mutual information developed in this paper reproduces the results obtained by the conventional real time formalism. The direct calculation reduces significantly the amount of computation.

Next, we consider whether the coefficient $C_{A B}^{(n)}$ is factorized or not. We computed $h^{(n)}(A, B) \equiv \frac{\left(C_{A B}^{(n)}\right)^{2}}{C_{A A}^{(n)} C_{B B}^{(n)}}$ in some cases. If $C_{A B}^{(n)}$ is factorized, $h^{(n)}(A, B)=1$. For $n=$ $1,2, \cdots, 20$, we obtained the result that $h^{(n)}(A, B)$ is almost equal to 1 when $A$ is a sphere and $B$ is a cube and when $A$ is a cube and $B$ is a cube in the center of which there is a cubic hollow and when $A$ is a cube in the center of which there is a cubic hollow and the shape of $B$ is the same as the shape of $A$ and the size of $B$ is different from the size of $A$. These results suggest that the coefficient $C_{A B}^{(n)}$ is factorized in a massless free scalar field in $(3+1)$ dimension. This factorization is not clear in the formal expressions in (4.7) and (4.15) and numerical calculation is important to check the factorization.

For $d=2$, we calculate numerically the (Rényi) mutual information $I^{(n)}(A, B)$ of two disks $A$ and $B$ whose radii are $R_{A}=R_{B} \equiv R$, and the distance between the centers of them is $r$ for $d=2$. We put the centers of the disks on a lattice. We define the disk as a set of points which are at distances of $\tilde{R}$ or less from the center of the disk. In order to reduce the effect of the discretization, we correct $\tilde{R}$ and define the radius of this disk as $R:=(S / \pi)^{1 / 2}$ where $S$ is a number of points in the disk. 


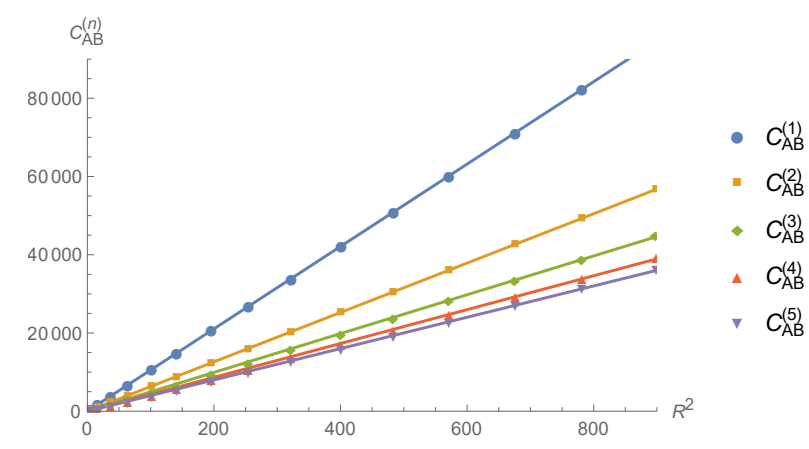

Figure 3. The coefficient $C_{A B}^{(n)}$ as a function of $R^{2}$, where $C_{A B}^{(n)}$ is the coefficient of the (Rényi) mutual information $I^{(n)}(A, B)$ of two disks $A$ and $B$ whose radii are $R_{A}=R_{B} \equiv R$. The lines are the best linear fit.

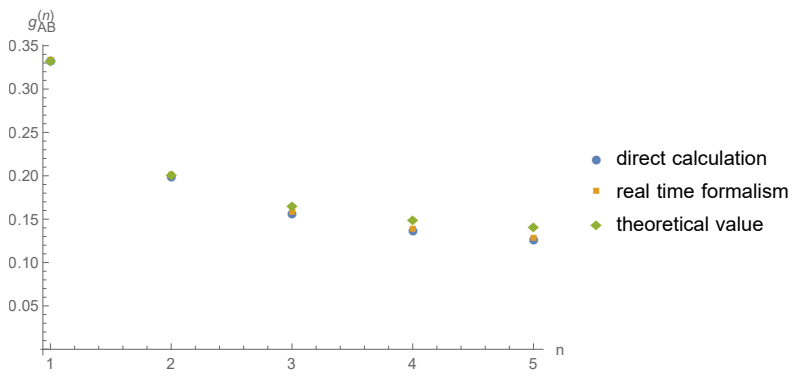

Figure 4. The coefficient $g_{A B}^{(n)}$ in (5.17) as a function of $n$. We show the values of $g_{A B}^{(n)}$ computed by the direct calculation developed in this paper and the real time formalism, and the theoretical values $\left.g_{A B}^{(n)}\right|_{d=2}$ in (5.18).

We show the computed values of $C_{A B}^{(n)}$ in (5.15) as a function of $R^{2}$ in figure 3. As can be seen, $C_{A B}^{(n)}$ is proportional to $R^{2}$.

Thus, we obtain

$$
I^{(n)}(A, B) \simeq g_{A B}^{(n)} \frac{R^{2}}{r^{2}}
$$

where $g_{A B}^{(n)}$ is a dimensionless constant. We calculate the coefficient $g_{A B}^{(n)}$ from slopes of graphs of $C_{A B}^{(n)}$ as a function of $R^{2}$. We show the computed values of $g_{A B}^{(n)}$ as a function of $n$ in figure 4.

For comparison, we also showed the values of $g_{A B}^{(n)}$ computed by the real time formalism [21] and the theoretical values,

$$
\begin{aligned}
\left.g_{A B}^{(n)}\right|_{d=2}= & \frac{n}{2 \pi^{2}(n-1)} \int_{0}^{\infty} \int_{0}^{\infty}\left(\frac{(1-x)(1-y)\left(1-(x y)^{n-1}\right)}{1-x y}+\left(1-x^{n-1}\right)\left(1-y^{n-1}\right)\right) \\
& \times \frac{d x d y}{(1+x)(1+y)\left(1-x^{n}\right)\left(1-y^{n}\right)},
\end{aligned}
$$

obtained by Cardy [28]. In order to calculate the coefficient $g_{A B}^{(n)}$ by the real time formalism, we plotted the (Rényi) mutual information $I^{(n)}(A, B)$ as a function of $r$ and extract the coefficient in the same way as the mutual information [26]. From figure 4, for $n=1$, 
the value of $g_{A B}^{(n)}$ computed by the direct calculation and one computed by the real time formalism and theoretical one are almost equal. For $n>1$, the values of $g_{A B}^{(n)}$ computed by the direct calculation and those computed by the real time formalism are almost equal, however, the theoretical values $\left.g_{A B}^{(n)}\right|_{d=2}$ are slightly larger than numerical computed values. The discrepancy for $n>1$ probably indicates that the effect of the discretization become important when $n$ increases. The direct calculation of the (Rényi) mutual information developed in this paper reproduces the results obtained by the conventional real time formalism. The direct calculation reduces significantly the amount of computation.

Next, we consider whether the coefficient $C_{A B}^{(n)}$ is factorized or not. We computed $h^{(n)}(A, B) \equiv \frac{\left(C_{A B}^{(n)}\right)^{2}}{C_{A A}^{(n)} C_{B B}^{(n)}}$ in some cases. If $C_{A B}^{(n)}$ is factorized, $h^{(n)}(A, B)=1$. For $n=$ $1,2, \cdots, 20$, we obtained the result that $h^{(n)}(A, B)$ is almost equal to 1 when $A$ is a disk and $B$ is a square and when $A$ is a square and $B$ is a square in the center of which there is a square hole and when $A$ is a square in the center of which there is a square hole and the shape of $B$ is the same as the shape of $A$ and the size of $B$ is different from the size of $A$. These results suggest that the coefficient $C_{A B}^{(n)}$ is factorized in a massless free scalar field in $(2+1)$ dimension. This factorization is not clear in the formal expressions in (4.7) and (4.15) and numerical calculation is important to check the factorization.

\section{Conclusion and discussions}

In this paper, we considered the (Rényi) mutual information, $I^{(n)}(A, B)=S_{A}^{(n)}+S_{B}^{(n)}-$ $S_{A \cup B}^{(n)}$, of distant compact spatial regions $A$ and $B$ in the vacuum state of a free scalar field. The distance $r$ between $A$ and $B$ is much greater than their sizes $R_{A, B}$ and the (Rényi) mutual information behaves as $I^{(n)}(A, B) \sim C_{A B}^{(n)}\langle 0|\phi(r) \phi(0)| 0\rangle^{2}$. We obtained the direct expression of $C_{A B}^{(n)}$ for arbitrary regions $A$ and $B$. We performed the analytical continuation of $n$ and obtain the mutual information $I(A, B)=\lim _{n \rightarrow 1} I^{(n)}(A, B)$. When $n=2,3, C_{A B}^{(n)}$ is not entangled, i.e. it is a simple product of functions each of which is determined by the shape of $A(B)$. For general $n, C_{A B}^{(n)}$ is not a simple product of functions each of which is determined by the shape of $A(B)$ and $C_{A B}^{(n)}$ is entangled. For example, $C_{A B}^{(n)}$ is entangled when $n=1,4$.

We applied our method to a massless free scalar field in $(d+1)$ dimension and performed numerical calculation for $d=2,3$. We showed that our method reproduce the results computed by conventional real time formalism. We showed that $C_{A B}^{(n)}$ is factorized in a $(d+1)$-dimensional massless free scalar field by numerical calculation for $d=2,3$. This factorization is not clear in the formal expressions in (4.7) and (4.15) and numerical calculation is important to check the factorization.

The direct expression is useful for the numerical computation. By using the direct expression, we can compute directly $I(A, B)$ without computing $S_{A}, S_{B}$ and $S_{A \cup B}$ respectively, so it reduces significantly the amount of computation.

It is an interesting future problem to apply our direct expression to study the shape dependence of $C_{A B}^{(n)}$. For example, the corner contribution to mutual information in $(2+1)$ dimension is an interesting problem. The corner contributions to entanglement entropy 
in $(2+1)$ dimension are universal and have important information of the QFT [32-35], however, the corner contribution to mutual information has not been studied well. Our method is useful for studying the corner contributions of mutual information. It is also an interesting future problem to generalize our method to the entanglement negativity [36, 37].

\section{Acknowledgments}

I would like to thank Tokiro Numasawa, Sotaro Sugishita, Tadashi Takayanagi, Kotaro Tamaoka, and Kento Watanabe for useful comments and discussions. I also thank the Yukawa Institute for Theoretical Physics at Kyoto University. Discussions during the workshop YITP-T-19-03 "Quantum Information and String Theory 2019" were useful. This work was supported by JSPS KAKENHI Grant Number JP19K14721.

\section{A Derivation of $0 \leq \lambda_{i}<1$}

We show that the range of the eigenvalues $\lambda_{i}$ of $Y$ in (3.16) is $0 \leq \lambda_{i}<1 . A$ and $D$ in (3.10) and (3.11) are positive definite symmetric matrices because $W$ and $W^{-1}$ are positive definite symmetric matrices. So, $X=\left(A^{-1}+D\right)^{-1 / 2}$ in $(3.15)$ is a positive definite symmetric matrix.

In order to show that $Y$ is a positive semidefinite matrix, we use the following identity,

$$
\left(\begin{array}{ll}
1 & 0 \\
0 & 1
\end{array}\right)=\left(\begin{array}{cc}
A & B \\
B^{T} & C
\end{array}\right)\left(\begin{array}{cc}
D & E \\
E^{T} & F
\end{array}\right)=\left(\begin{array}{cc}
A D+B E^{T} & A E+B F \\
B^{T} D+C E^{T} & B^{T} E+C F
\end{array}\right) .
$$

From (A.1), we obtain $D A-1=-E B^{T}$ and $B^{T}=-F^{-1} E^{T} A$. Thus we rewrite $D-A^{-1}$ in $Y$ as

$$
D-A^{-1}=(D A-1) A^{-1}=-E B^{T} A^{-1}=E F^{-1} E^{T} .
$$

Because $F^{-1}$ is a positive definite matrix and (A.2), $D-A^{-1}$ is a positive semidefinite matrix. Therefore, $Y=X\left(D-A^{-1}\right) X$ is a positive semidefinite matrix and we obtain $0 \leq \lambda_{i}$.

Next we consider the upper bound of $\lambda_{i}$. We rewrite $1-Y$ as

$$
\begin{aligned}
1-Y & =\left(A^{-1}+D\right)^{-1 / 2}\left[A^{-1}+D-\left(D-A^{-1}\right)\right]\left(A^{-1}+D\right)^{-1 / 2} \\
& =\left(A^{-1}+D\right)^{-1 / 2} 2 A^{-1}\left(A^{-1}+D\right)^{-1 / 2}
\end{aligned}
$$

Because $A^{-1}$ is a positive definite matrix and (A.3), $1-Y$ is a positive definite matrix and we obtain $\lambda_{i}<1$. Therefore, we have shown $0 \leq \lambda_{i}<1$.

\section{B The calculation of $F(n, a, b)$ in (4.6)}

We calculate the summation $F(n, a, b)$ in (4.6) for $0 \leq a<1,0 \leq b<1$. We expand $\left(1-\cos \left(\frac{2 \pi k}{n}\right)\right)^{2}$ in $(4.6)$ and rewrite $F(n, a, b)$ as

$$
\begin{aligned}
F(n, a, b)= & \frac{n}{a b}+\frac{1}{a}\left(1-\frac{1}{b}\right) \sum_{k=0}^{n-1} \frac{1}{1-b \cos \left(\frac{2 \pi k}{n}\right)}+\frac{1}{b}\left(1-\frac{1}{a}\right) \sum_{k=0}^{n-1} \frac{1}{1-a \cos \left(\frac{2 \pi k}{n}\right)} \\
& +\left(1-\frac{1}{a}\right)\left(1-\frac{1}{b}\right) \sum_{k=0}^{n-1} \frac{1}{1-a \cos \left(\frac{2 \pi k}{n}\right)} \frac{1}{1-b \cos \left(\frac{2 \pi k}{n}\right)} .
\end{aligned}
$$


In order to calculate the summations in (B.1), we use the following expansion,

$$
\frac{1}{1-a \cos \theta}=\left(1+\rho(a)^{2}\right) \frac{1}{1-\rho(a) e^{i \theta}} \frac{1}{1-\rho(a) e^{-i \theta}}=\left(1+\rho(a)^{2}\right) \sum_{p=0}^{\infty} \sum_{p^{\prime}=0}^{\infty} \rho(a)^{p+p^{\prime}} e^{i\left(p-p^{\prime}\right) \theta}
$$

where

$$
\rho(a) \equiv \frac{1}{a}\left(1-\sqrt{1-a^{2}}\right),
$$

here $0 \leq \rho(a)<1$ for $0 \leq a<1$, and

$$
a=\frac{2 \rho(a)}{1+\rho(a)^{2}} .
$$

The expansion (B.2) in the limit $a \rightarrow 1-$ was used in [28].

\section{B.1 The calculation of $\sum_{k=0}^{n-1} \frac{1}{1-a \cos \left(\frac{2 \pi k}{n}\right)}$}

By using the expansion in (B.2), we obtain

$$
\sum_{k=0}^{n-1} \frac{1}{1-a \cos \left(\frac{2 \pi k}{n}\right)}=\left(1+\rho(a)^{2}\right) \sum_{p=0}^{\infty} \sum_{p^{\prime}=0}^{\infty} \rho(a)^{p+p^{\prime}} \sum_{k=0}^{n-1} e^{i\left(p-p^{\prime}\right) \frac{2 \pi k}{n}} .
$$

We split the $p, p^{\prime}$ summation into three parts,

$$
\sum_{p=0}^{\infty} \sum_{p^{\prime}=0}^{\infty}=\left.\sum_{p^{\prime}=0}^{\infty} \sum_{l=0}^{\infty}\right|_{p=p^{\prime}+l}+\left.\sum_{p=0}^{\infty} \sum_{l=0}^{\infty}\right|_{p^{\prime}=p+l}-\left.\sum_{p=0}^{\infty}\right|_{p^{\prime}=p}
$$

where we subtracted the $p=p^{\prime}$ part to avoid double counting. From (B.5) and (B.6), we obtain

$$
\begin{aligned}
\sum_{p=0}^{\infty} \sum_{p^{\prime}=0}^{\infty} \rho(a)^{p+p^{\prime}} & \sum_{k=0}^{n-1} e^{i\left(p-p^{\prime}\right) \frac{2 \pi k}{n}} \\
& =\sum_{p^{\prime}=0}^{\infty} \sum_{l=0}^{\infty} \rho^{2 p^{\prime}+l} \sum_{k=0}^{n-1} e^{i l \frac{2 \pi k}{n}}+\sum_{p=0}^{\infty} \sum_{l=0}^{\infty} \rho^{2 p+l} \sum_{k=0}^{n-1} e^{-i l \frac{2 \pi k}{n}}-\sum_{p=0}^{\infty} \rho^{2 p} \sum_{k=0}^{n-1} 1 \\
& =n \sum_{p^{\prime}=0}^{\infty} \sum_{j=0}^{\infty} \rho^{2 p^{\prime}+n j}+n \sum_{p=0}^{\infty} \sum_{j=0}^{\infty} \rho^{2 p+n j}-n \sum_{p=0}^{\infty} \rho^{2 p} \\
& =\frac{2 n}{\left(1-\rho^{2}\right)\left(1-\rho^{n}\right)}-\frac{n}{1-\rho^{2}}=\frac{n}{1-\rho^{2}} \cdot \frac{1+\rho^{n}}{1-\rho^{n}}
\end{aligned}
$$

where we have used

$$
\sum_{k=0}^{n-1} e^{i l \frac{2 \pi k}{n}}=n \delta_{l, n j} \quad(j=0,1,2, \cdots) .
$$

We substitute (B.7) into (B.5) and obtain

$$
\sum_{k=0}^{n-1} \frac{1}{1-a \cos \left(\frac{2 \pi k}{n}\right)}=\left(1+\rho(a)^{2}\right) \frac{n}{1-\rho(a)^{2}} \cdot \frac{1+\rho(a)^{n}}{1-\rho(a)^{n}} .
$$




\section{B.2 The calculation of $\sum_{k=0}^{n-1} \frac{1}{1-a \cos \left(\frac{2 \pi k}{n}\right)} \frac{1}{1-b \cos \left(\frac{2 \pi k}{n}\right)}$}

In the same way as above, by using the expansion in (B.2), we obtain

$$
\begin{aligned}
& \sum_{k=0}^{n-1} \frac{1}{1-a \cos \left(\frac{2 \pi k}{n}\right)} \frac{1}{1-b \cos \left(\frac{2 \pi k}{n}\right)} \\
& =\left(1+\rho(a)^{2}\right)\left(1+\rho(b)^{2}\right) \sum_{k=0}^{n-1} \sum_{p=0}^{\infty} \sum_{p^{\prime}=0}^{\infty} \rho(a)^{p+p^{\prime}} e^{i\left(p-p^{\prime}\right) \frac{2 \pi k}{n}} \sum_{q=0}^{\infty} \sum_{q^{\prime}=0}^{\infty} \rho(b)^{q+q^{\prime}} e^{i\left(q-q^{\prime}\right) \frac{2 \pi k}{n}} .
\end{aligned}
$$

From (B.6), we can rewrite the summations in (B.10) as

$$
\begin{aligned}
\sum_{p=0}^{\infty} \sum_{p^{\prime}=0}^{\infty} \sum_{q=0}^{\infty} \sum_{q^{\prime}=0}^{\infty}= & \left(\left.\sum_{p^{\prime}=0}^{\infty} \sum_{l=0}^{\infty}\right|_{p=p^{\prime}+l}+\left.\sum_{p=0}^{\infty} \sum_{l=0}^{\infty}\right|_{p^{\prime}=p+l}-\left.\sum_{p=0}^{\infty}\right|_{p^{\prime}=p}\right) \\
& \times\left(\left.\sum_{q^{\prime}=0}^{\infty} \sum_{m=0}^{\infty}\right|_{q=q^{\prime}+m}+\left.\sum_{q=0}^{\infty} \sum_{m=0}^{\infty}\right|_{q^{\prime}=q+m}-\left.\sum_{q=0}^{\infty}\right|_{q^{\prime}=q}\right) .
\end{aligned}
$$

We substitute (B.11) into (B.10) and obtain

$$
\begin{gathered}
\frac{1}{\left(1+\rho(a)^{2}\right)\left(1+\rho(b)^{2}\right)} \sum_{k=0}^{n-1} \frac{1}{1-a \cos \left(\frac{2 \pi k}{n}\right)} \frac{1}{1-b \cos \left(\frac{2 \pi k}{n}\right)} \\
=\sum_{k=0}^{n-1}\left[2 \left(\sum_{p^{\prime}=0}^{\infty} \sum_{l=0}^{\infty} \sum_{q^{\prime}=0}^{\infty} \sum_{m=0}^{\infty} \rho(a)^{2 p^{\prime}+l} \rho(b)^{2 q^{\prime}+m} e^{i(l+m) 2 \pi k / n}\right.\right. \\
+\sum_{p^{\prime}=0}^{\infty} \sum_{l=0}^{\infty} \sum_{q=0}^{\infty} \sum_{m=0}^{\infty} \rho(a)^{2 p^{\prime}+l} \rho(b)^{2 q+m} e^{i(l-m) 2 \pi k / n} \\
-\sum_{p^{\prime}=0}^{\infty} \sum_{l=0}^{\infty} \sum_{q=0}^{\infty} \rho(a)^{2 p^{\prime}+l} \rho(b)^{2 q} e^{i l 2 \pi k / n} \\
\left.-\sum_{p=0}^{\infty} \sum_{q^{\prime}=0}^{\infty} \sum_{m=0}^{\infty} \rho(a)^{2 p} \rho(b)^{2 q^{\prime}+m} e^{i m 2 \pi k / n}\right) \\
\left.+\sum_{p=0}^{\infty} \sum_{q=0}^{\infty} \rho(a)^{2 p} \rho(b)^{2 q}\right] .
\end{gathered}
$$

The last term in (B.12) can be evaluated as

$$
\sum_{k=0}^{n-1} \sum_{p=0}^{\infty} \sum_{q=0}^{\infty} \rho(a)^{2 p} \rho(b)^{2 q}=n \frac{1}{1-\rho(a)^{2}} \frac{1}{1-\rho(b)^{2}} .
$$

The third term in (B.12) can be evaluated as

$$
\begin{aligned}
\sum_{k=0}^{n-1} \sum_{p^{\prime}=0}^{\infty} \sum_{l=0}^{\infty} \sum_{q=0}^{\infty} \rho(a)^{2 p^{\prime}+l} \rho(b)^{2 q} e^{i l 2 \pi k / n} & =n \sum_{p^{\prime}=0}^{\infty} \sum_{j=0}^{\infty} \sum_{q=0}^{\infty} \rho(a)^{2 p^{\prime}+n j} \rho(b)^{2 q} \\
& =n \frac{1}{1-\rho(a)^{2}} \frac{1}{1-\rho(a)^{n}} \frac{1}{1-\rho(b)^{2}}
\end{aligned}
$$


The fourth term in (B.12) is obtained by interchanging $a$ and $b$ in the third term in (B.12).

We perform the $p^{\prime}$ and $q$ summations in the second term in (B.12) and obtain

$$
\begin{aligned}
\sum_{k=0}^{n-1} \sum_{p^{\prime}=0}^{\infty} \sum_{l=0}^{\infty} \sum_{q=0}^{\infty} \sum_{m=0}^{\infty} \rho(a)^{2 p^{\prime}+l} \rho(b)^{2 q+m} e^{i(l-m) 2 \pi k / n} \\
\quad=\frac{1}{1-\rho(a)^{2}} \frac{1}{1-\rho(b)^{2}} \sum_{k=0}^{n-1} \sum_{l=0}^{\infty} \sum_{m=0}^{\infty} \rho(a)^{l} \rho(b)^{m} e^{i(l-m) 2 \pi k / n}
\end{aligned}
$$

By using (B.6), we obtain

$$
\begin{aligned}
& \sum_{k=0}^{n-1} \sum_{l=0}^{\infty} \sum_{m=0}^{\infty} \rho(a)^{l} \rho(b)^{m} e^{i(l-m) 2 \pi k / n} \\
& =\sum_{k=0}^{n-1}\left[\sum_{l=0}^{\infty} \sum_{\alpha=0}^{\infty} \rho(a)^{l} \rho(b)^{l+\alpha} e^{-i \alpha 2 \pi k / n}+\sum_{m=0}^{\infty} \sum_{\alpha=0}^{\infty} \rho(a)^{m+\alpha} \rho(b)^{m} e^{i \alpha 2 \pi k / n}-\sum_{l=0}^{\infty} \rho(a)^{l} \rho(b)^{l}\right] \\
& =n \sum_{l=0}^{\infty} \sum_{j=0}^{\infty} \rho(a)^{l} \rho(b)^{l+n j}+n \sum_{m=0}^{\infty} \sum_{j=0}^{\infty} \rho(a)^{m+n j} \rho(b)^{m}-n \frac{1}{1-\rho(a) \rho(b)} \\
& =\frac{n}{1-\rho(a) \rho(b)}\left[\frac{1}{1-\rho(b)^{n}}+\frac{1}{1-\rho(a)^{n}}-1\right]
\end{aligned}
$$

Thus, we substitute (B.16) into (B.15) and obtain the second term in (B.12)

$$
\begin{aligned}
& \sum_{k=0}^{n-1} \sum_{p^{\prime}=0}^{\infty} \sum_{l=0}^{\infty} \sum_{q=0}^{\infty} \sum_{m=0}^{\infty} \rho(a)^{2 p^{\prime}+l} \rho(b)^{2 q+m} e^{i(l-m) 2 \pi k / n} \\
& \quad=\frac{1}{1-\rho(a)^{2}} \frac{1}{1-\rho(b)^{2}} \frac{n}{1-\rho(a) \rho(b)}\left[\frac{1}{1-\rho(a)^{n}}+\frac{1}{1-\rho(b)^{n}}-1\right]
\end{aligned}
$$

We perform the $p^{\prime}$ and $q^{\prime}$ summations in the first term in (B.12) and obtain

$$
\begin{aligned}
& \sum_{k=0}^{n-1} \sum_{p^{\prime}=0}^{\infty} \sum_{l=0}^{\infty} \sum_{q^{\prime}=0}^{\infty} \sum_{m=0}^{\infty} \rho(a)^{2 p^{\prime}+l} \rho(b)^{2 q^{\prime}+m} e^{i(l+m) 2 \pi k / n} \\
& \quad=\frac{1}{1-\rho(a)^{2}} \frac{1}{1-\rho(b)^{2}} \sum_{k=0}^{n-1} \sum_{l=0}^{\infty} \sum_{m=0}^{\infty} \rho(a)^{l} \rho(b)^{m} e^{i(l+m) 2 \pi k / n}
\end{aligned}
$$

By using (B.8), we obtain

$$
\begin{aligned}
& \sum_{k=0}^{n-1} \sum_{l=0}^{\infty} \sum_{m=0}^{\infty} \rho(a)^{l} \rho(b)^{m} e^{i(l+m) 2 \pi k / n}=n \sum_{j=0}^{\infty} \sum_{l=0}^{n j} \rho(a)^{l} \rho(b)^{n j-l} \\
& =n \sum_{j=0}^{\infty} \rho(b)^{n j}\left(\frac{1-(\rho(a) / \rho(b))^{n j+1}}{1-(\rho(a) / \rho(b))}\right)=\frac{n}{\rho(b)-\rho(a)}\left[\frac{\rho(b)}{1-\rho(b)^{n}}-\frac{\rho(a)}{1-\rho(a)^{n}}\right] .
\end{aligned}
$$


We substitute (B.19) into (B.18) and obtain the first term in (B.12)

$$
\begin{aligned}
& \sum_{k=0}^{n-1} \sum_{p^{\prime}=0}^{\infty} \sum_{l=0}^{\infty} \sum_{q^{\prime}=0}^{\infty} \sum_{m=0}^{\infty} \rho(a)^{2 p^{\prime}+l} \rho(b)^{2 q^{\prime}+m} e^{i(l+m) 2 \pi k / n} \\
& \quad=\frac{1}{1-\rho(a)^{2}} \frac{1}{1-\rho(b)^{2}} \frac{n}{\rho(b)-\rho(a)}\left[\frac{\rho(b)}{1-\rho(b)^{n}}-\frac{\rho(a)}{1-\rho(a)^{n}}\right] .
\end{aligned}
$$

Finally, we substitute (B.13), (B.14), (B.17) and (B.20) into (B.12) and obtain

$$
\begin{aligned}
\frac{1}{\left(1+\rho(a)^{2}\right)\left(1+\rho(b)^{2}\right)} \sum_{k=0}^{n-1} \frac{1}{1-a \cos \left(\frac{2 \pi k}{n}\right)} \frac{1}{1-b \cos \left(\frac{2 \pi k}{n}\right)} \\
=2\left[\frac{1}{1-\rho(a)^{2}} \frac{1}{1-\rho(b)^{2}} \frac{n}{\rho(b)-\rho(a)}\left(\frac{\rho(b)}{1-\rho(b)^{n}}-\frac{\rho(a)}{1-\rho(a)^{n}}\right)\right. \\
\quad+\frac{1}{1-\rho(a)^{2}} \frac{1}{1-\rho(b)^{2}} \frac{n}{1-\rho(a) \rho(b)}\left(\frac{1}{1-\rho(a)^{n}}+\frac{1}{1-\rho(b)^{n}}-1\right) \\
\left.\quad-n \frac{1}{1-\rho(a)^{2}} \frac{1}{1-\rho(b)^{2}}\left(\frac{1}{1-\rho(a)^{n}}+\frac{1}{1-\rho(b)^{n}}\right)\right] \\
+n \frac{1}{1-\rho(a)^{2}} \frac{1}{1-\rho(b)^{2}} \\
=2 n \frac{1}{1-\rho(a)^{2}} \frac{1}{1-\rho(b)^{2}} \frac{1}{\rho(a)-\rho(b)} \frac{1}{1-\rho(a) \rho(b)} \\
\times\left[\frac{\rho(a)\left(1-\rho(b)^{2}\right)}{1-\rho(a)^{n}}-\frac{\rho(b)\left(1-\rho(a)^{2}\right)}{1-\rho(b)^{n}}-\frac{1}{2}(\rho(a)-\rho(b))(1+\rho(a) \rho(b))\right] .
\end{aligned}
$$

We substitute (B.9) and (B.21) into (B.1) and obtain

$$
\begin{gathered}
F(n, a, b)=n \frac{\left(1+p^{2}\right)\left(1+q^{2}\right)}{4 p q}\left[2-\frac{(1-p)\left(1+p^{n}\right)}{(1+p)\left(1-p^{n}\right)}-\frac{(1-q)\left(1+q^{n}\right)}{(1+q)\left(1-q^{n}\right)}\right. \\
+\frac{2}{(1+p)(1+q)(p-q)(1-p q)}\left\{\frac{1-p}{1-p^{n}} p(1-q)^{2}(1+q)-\frac{1-q}{1-q^{n}} q(1-p)^{2}(1+p)\right. \\
-(p-q)(1+p q-(p+q) p q)\}]
\end{gathered}
$$

where $0 \leq a<1,0 \leq b<1$ and

$$
p \equiv \rho(a)=\frac{1}{a}\left(1-\sqrt{1-a^{2}}\right), q \equiv \rho(b)=\frac{1}{b}\left(1-\sqrt{1-b^{2}}\right) .
$$

Thus (B.22) is equal to (4.7).

Open Access. This article is distributed under the terms of the Creative Commons Attribution License (CC-BY 4.0), which permits any use, distribution and reproduction in any medium, provided the original author(s) and source are credited. 


\section{References}

[1] S. Ryu and T. Takayanagi, Holographic derivation of entanglement entropy from AdS/CFT, Phys. Rev. Lett. 96 (2006) 181602 [hep-th/0603001] [INSPIRE].

[2] S. Ryu and T. Takayanagi, Aspects of holographic entanglement entropy, JHEP 08 (2006) 045 [hep-th/0605073] [INSPIRE].

[3] T. Faulkner, A. Lewkowycz and J. Maldacena, Quantum corrections to holographic entanglement entropy, JHEP 11 (2013) 074 [arXiv:1307.2892] [INSPIRE].

[4] B. Swingle, Entanglement renormalization and holography, Phys. Rev. D 86 (2012) 065007 [arXiv: 0905.1317] [INSPIRE].

[5] M. Nozaki, S. Ryu and T. Takayanagi, Holographic geometry of entanglement renormalization in quantum field theories, JHEP 10 (2012) 193 [arXiv: 1208.3469] [INSPIRE].

[6] M. Miyaji and T. Takayanagi, Surface/state correspondence as a generalized holography, PTEP 2015 (2015) 073B03 [arXiv: 1503.03542] [INSPIRE].

[7] P. Caputa, M. Miyaji, T. Takayanagi and K. Umemoto, Holographic entanglement of purification from conformal field theories, Phys. Rev. Lett. 122 (2019) 111601 [arXiv: 1812.05268] [INSPIRE].

[8] N. Shiba and T. Takayanagi, Volume law for the entanglement entropy in non-local QFTs, JHEP 02 (2014) 033 [arXiv:1311.1643] [INSPIRE].

[9] A. Mollabashi, N. Shiba and T. Takayanagi, Entanglement between two interacting CFTs and generalized holographic entanglement entropy, JHEP 04 (2014) 185 [arXiv:1403.1393] [INSPIRE].

[10] M. Miyaji, T. Numasawa, N. Shiba, T. Takayanagi and K. Watanabe, Continuous multiscale entanglement renormalization ansatz as holographic surface-state correspondence, Phys. Rev. Lett. 115 (2015) 171602 [arXiv: 1506. 01353] [INSPIRE].

[11] M. Miyaji, T. Numasawa, N. Shiba, T. Takayanagi and K. Watanabe, Distance between quantum states and gauge-gravity duality, Phys. Rev. Lett. 115 (2015) 261602 [arXiv: 1507.07555] [INSPIRE].

[12] T. Miyagawa, N. Shiba and T. Takayanagi, Double-trace deformations and entanglement entropy in AdS, Fortsch. Phys. 64 (2016) 92 [arXiv:1511.07194] [INSPIRE].

[13] T. Numasawa, N. Shiba, T. Takayanagi and K. Watanabe, EPR pairs, local projections and quantum teleportation in holography, JHEP 08 (2016) 077 [arXiv: 1604.01772] [INSPIRE].

[14] M. Levin and X.-G. Wen, Detecting topological order in a ground state wave function, Phys. Rev. Lett. 96 (2006) 110405 [cond-mat/0510613] [INSPIRE].

[15] A. Kitaev and J. Preskill, Topological entanglement entropy, Phys. Rev. Lett. 96 (2006) 110404 [hep-th/0510092] [INSPIRE].

[16] P. Calabrese and J.L. Cardy, Entanglement entropy and quantum field theory, J. Stat. Mech. 0406 (2004) P06002 [hep-th/0405152] [INSPIRE].

[17] N. Shiba, Aharonov-Bohm effect on entanglement entropy in conformal field theory, Phys. Rev. D 96 (2017) 065016 [arXiv: 1701.00688] [INSPIRE].

[18] S. Ghosh, R.M. Soni and S.P. Trivedi, On the entanglement entropy for gauge theories, JHEP 09 (2015) 069 [arXiv: 1501.02593] [INSPIRE].

[19] S. Aoki, T. Iritani, M. Nozaki, T. Numasawa, N. Shiba and H. Tasaki, On the definition of entanglement entropy in lattice gauge theories, JHEP 06 (2015) 187 [arXiv:1502.04267] [INSPIRE]. 
[20] Y. Nakai, N. Shiba and M. Yamada, Entanglement entropy and decoupling in the universe, Phys. Rev. D 96 (2017) 123518 [arXiv:1709.02390] [INSPIRE].

[21] L. Bombelli, R.K. Koul, J. Lee and R.D. Sorkin, A quantum source of entropy for black holes, Phys. Rev. D 34 (1986) 373 [inSPIRE].

[22] M. Srednicki, Entropy and area, Phys. Rev. Lett. 71 (1993) 666 [hep-th/9303048] [INSPIRE].

[23] L. Susskind and J. Uglum, Black hole entropy in canonical quantum gravity and superstring theory, Phys. Rev. D 50 (1994) 2700 [hep-th/9401070] [InSPIRE].

[24] D.N. Kabat, Black hole entropy and entropy of entanglement, Nucl. Phys. B 453 (1995) 281 [hep-th/9503016] [INSPIRE].

[25] N. Shiba, Entanglement entropy of two black holes and entanglement entropic force, Phys. Rev. D 83 (2011) 065002 [arXiv: 1011.3760] [INSPIRE].

[26] N. Shiba, Entanglement entropy of two spheres, JHEP 07 (2012) 100 [arXiv:1201.4865] [INSPIRE].

[27] M. Creutz, Quarks, gluons and lattices, Cambridge University Press, Cambridge, U.K. (1985).

[28] J. Cardy, Some results on the mutual information of disjoint regions in higher dimensions, J. Phys. A 46 (2013) 285402 [arXiv: 1304.7985] [InSPIRE].

[29] N. Shiba, Entanglement entropy of disjoint regions in excited states: an operator method, JHEP 12 (2014) 152 [arXiv: 1408.0637] [INSPIRE].

[30] P. Calabrese, J. Cardy and E. Tonni, Entanglement entropy of two disjoint intervals in conformal field theory II, J. Stat. Mech. 1101 (2011) P01021 [arXiv:1011.5482] [InSPIRE].

[31] M. Headrick, Entanglement Rényi entropies in holographic theories, Phys. Rev. D 82 (2010) 126010 [arXiv: 1006.0047] [INSPIRE].

[32] H. Casini and M. Huerta, Universal terms for the entanglement entropy in $2+1$ dimensions, Nucl. Phys. B $\mathbf{7 6 4}$ (2007) 183 [hep-th/0606256] [INSPIRE].

[33] H. Casini, M. Huerta and L. Leitao, Entanglement entropy for a Dirac fermion in three dimensions: vertex contribution, Nucl. Phys. B 814 (2009) 594 [arXiv:0811.1968] [INSPIRE].

[34] T. Hirata and T. Takayanagi, AdS/CFT and strong subadditivity of entanglement entropy, JHEP 02 (2007) 042 [hep-th/0608213] [INSPIRE].

[35] E. Fradkin and J.E. Moore, Entanglement entropy of $2 D$ conformal quantum critical points: hearing the shape of a quantum drum, Phys. Rev. Lett. 97 (2006) 050404 [cond-mat/0605683] [INSPIRE].

[36] G. Vidal and R.F. Werner, Computable measure of entanglement, Phys. Rev. A 65 (2002) 032314 [quant-ph/0102117] [INSPIRE].

[37] P. Calabrese, J. Cardy and E. Tonni, Entanglement negativity in quantum field theory, Phys. Rev. Lett. 109 (2012) 130502 [arXiv:1206.3092] [INSPIRE].

[38] C. Agón and T. Faulkner, Quantum corrections to holographic mutual information, JHEP 08 (2016) 118 [arXiv: 1511.07462] [INSPIRE].

[39] C.A. Agón, I. Cohen-Abbo and H.J. Schnitzer, Large distance expansion of mutual information for disjoint disks in a free scalar theory, JHEP 11 (2016) 073 [arXiv: 1505.03757] [INSPIRE].

[40] H.J. Schnitzer, Mutual Rényi information for two disjoint compound systems, arXiv: 1406.1161 [INSPIRE]. 